\title{
COMPARISON OF GEOMETRIC CORRECTION SCHEMES FOR GEOSTATIONARY OCEAN COLOR IMAGER SLOTS WITHOUT GCPS
}

\author{
J.Son ${ }^{1}$, H.Kim ${ }^{2}$, T.Kim ${ }^{1} *$ \\ ${ }^{1}$ Dept. of Geoinformatic Engineering, Inha University, 100 Inharo, Namgu, Incheon, Republic of Korea \\ - json8520@gmail.com, tezid@inha.ac.kr \\ 2 3DLabs Co., Ltd., 100 Inharo, Namgu, Incheon, Korea - khanai91@gmail.com
}

Commission IV, ICWG IV/III

KEY WORDS: GOCI, Geometric correction, Satellite image, GCP, Sensor Model

\begin{abstract}
:
Currently, the geometric correction process of GOCI (Geostationary Ocean Color Imager) image is performed by matching slot images against shorelines and utilizing the matching results as GCPs (Ground Control Point). However, there are several GOCI slots without shorelines and for such slots acquiring GCPs is not easy. The purpose of this paper is to compare several alternative geometric correction schemes applicable to the slots without GCPs. We analyzed three schemes. The first scheme is to apply the correction angle of the same slot in the most recent dataset. The second scheme is to apply correction angle of the previous slot in the current dataset. And the last scheme is to apply correction angle of the slot with the largest number of GCPs in the same time dataset. Overall process for comparing the quality of the three geometric correction schemes consisted of the following steps. Firstly, using ephemeris metadata of GOCI Level 1A, we established initial sensor model, which defines geometry relationship between ground coordinate system and image coordinate system of a GOCI image. And then, by matching edge detected from GOCI slot images and shoreline landmark chips, we obtained GCPs. Using these GCPs, we calculated correction angle of each slot. After then, through the three schemes, we conducted precision sensor modeling. Among three schemes, geometric correction applying the previous slot correction angle showed the best quality. The average RMSE of this scheme was about $1.4 \mathrm{~km}$, which was quite close to geometric correction quality applying correction angles from GCPs.
\end{abstract}

\section{INTRODUCTION}

GOCI (Geostationary Ocean Color Imager) instrument is s payload of COMS (Communication, Ocean, and Meteorological Satellite) developed to perform marine environmental observation missions. GOCI image data are acquired every 1 hour during Korea daytime. One GOCI image consists of 16 slot images, which cover an area of $2500 \mathrm{~km}$ x $2500 \mathrm{~km}$ centered on the Korean peninsula. Each slot image has multispectral images of 8 channels. GOCI image slots contain geometric distortion due to Earth rotation, curvature, and satellite position and attitude measurement error. In order to utilize the GOCI satellite image data, we should correct these geometric distortions and mosaic the 16 slots into one scene through geometric correction process (Kim, 2018).

Currently, the geometric correction process of GOCI image is performed by matching image slots against shorelines and utilizing the matching results as GCPs (Ground Control Points) (Yang and Song, 2012). However, there are several GOCI slots without shorelines within their imaging area and for such slots acquiring GCPs are not easy. Besides clouds often occlude shorelines. As shown in the figure 1, there is little or no shoreline in the slot $1,2,13,14$, and 15 . GCPs cannot be obtained for those slots and alternative geometric correction schemes are required.

Therefore, we propose three alternative geometric correction schemes applicable to the GOCI slots without GCPs. And using these schemes, we perform geometric correction and analyze a quality of the results.

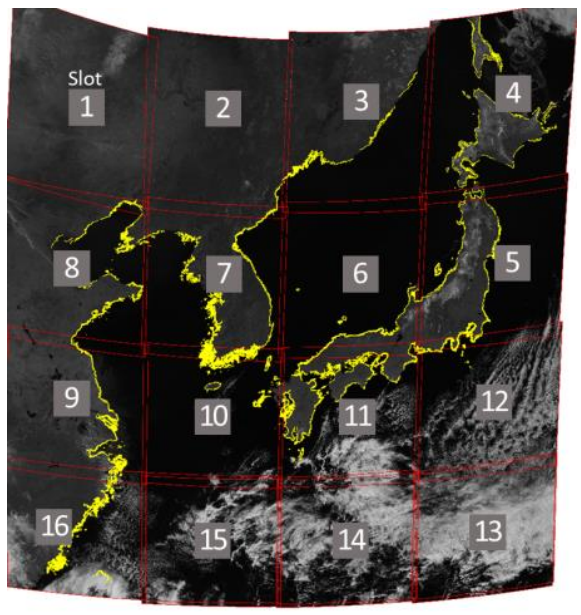

Figure 1. GOCI slots arrangement

\section{PROPOSED METHOD}

Currently, the geometric correction process of GOCI satellite image data is performed in four steps: initial sensor modeling, landmark chip matching, precision sensor modeling, image resampling (Yang and Song, 2012). In the initial sensor modeling geometric model is established using ephemeris information provided by satellite to define the relationship between the ground coordinate system and image coordinate system of each GOCI slot image. Note that accurate geometric 
relationship is yet to be obtained by the initial sensor model due to the error in the ephemeris information. Therefore, using GCPs, initial sensor model should be corrected. These GCPs are obtained by landmark chip matching. Each landmark chip is composed of shoreline image and ground coordinates. Through matching landmark chip and GOCI slot image, image coordinates can be obtained that corresponding ground coordinates of landmark chip. From this matching result, we can obtain GCPs (Lee et al., 2005). Using these GCPs, correction angle is calculated. Precision sensor model is obtained by applying rotation matrix of the correction angle to the initial sensor model. Finally in the image resampling, The GOCI slot images are relocated according to the ground coordinates.

In these processes, primary data for correcting geometric distortion is GCP. However, some slots contain little or no shorelines and acquiring GCPs are not easy. Consequently, these slots are not able to have their own correction angle. For these cases, we proposed three altermatic geometric correction schemes.

\subsection{Alternative Geometric Correction Schemes}

2.1.1 Scheme 1: The first scheme is to apply the correction angle of the same slot in the dataset taken most recently. This is the scheme that is currently applied within GOCI image generation system.

Since the GOCI is a payload of a geostationary satellite, it can be assumed that the geometry of a GOCI image at image acquisition is almost unchanged. Therefore, we may use the correction angle of the same slot in the most recent dataset

However, for slot 14, the shoreline does not exist at all in every dataset. Therefore, we were not able to use this scheme for slot 14. Geometric correction of slot 14 should be performed only with the initial sensor model.

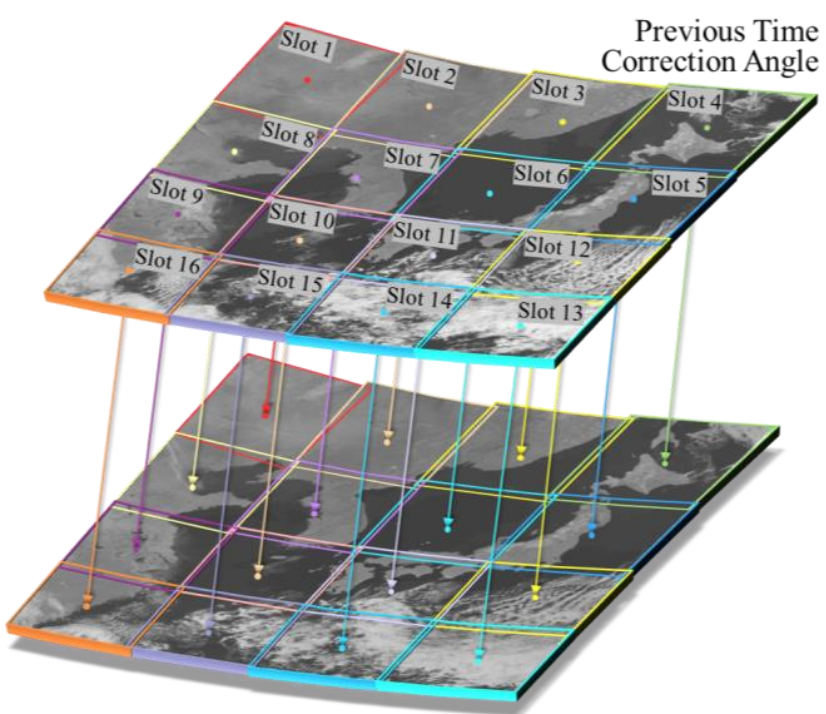

Figure 2. Using previous time correction angle

2.1.2 Scheme 2: The second scheme is to apply correction angle of the previous slot in the same dataset.

If a variation of geometry between slots is smaller than that between datasets, geometric correction with the correction angle of the previous slot in the same time dataset. can show better quality.
By default, this scheme applied the correction angle of the previous slot. If the previous slot also does not have its own correction angle, we used the correction angle of the closest to the previous slots. Figure 3 show the imaging sequence of the GOCI slots.

In case of slot 1 , since there is no previous slot, the correction angle of slot 2 was applied.

\section{Slot Imaging Sequence}

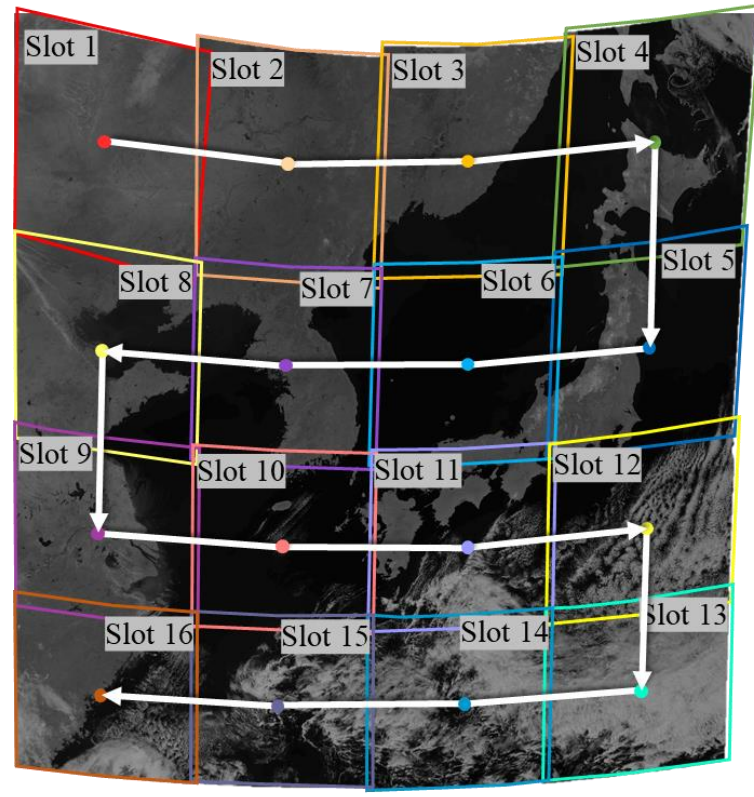

Figure 3. GOCI slot imaging sequence

2.1.3 Scheme 3: The last scheme is to apply correction angle of the slot with the largest number of GCPs in the same dataset. In scheme 1 or scheme 2 , the error of reused correction angle was often amplified. Therefore, a correction angle with the best performance can be tried alternatively.

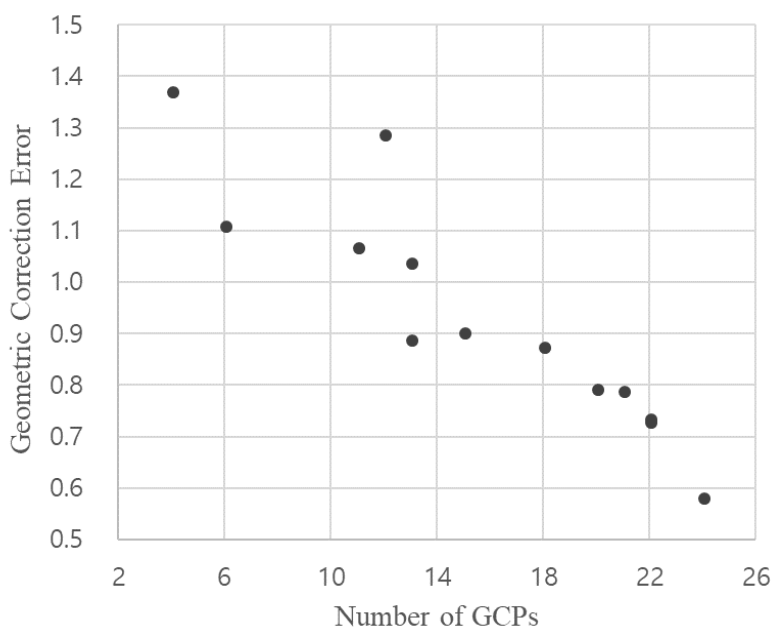

Figure 4. Relationship between number of GCPs and geometric correction error

As shown in Figure 4, the larger number of GCPs used for the correction angle calculation, the smaller geometric correction error. This showed that the number of GCPs was related to the performance of the correction angle. Accordingly, we 
performed geometric correction with correction angle of the slot with the largest number of GCPs in the same time data.

\subsection{Quality Comparison Process}

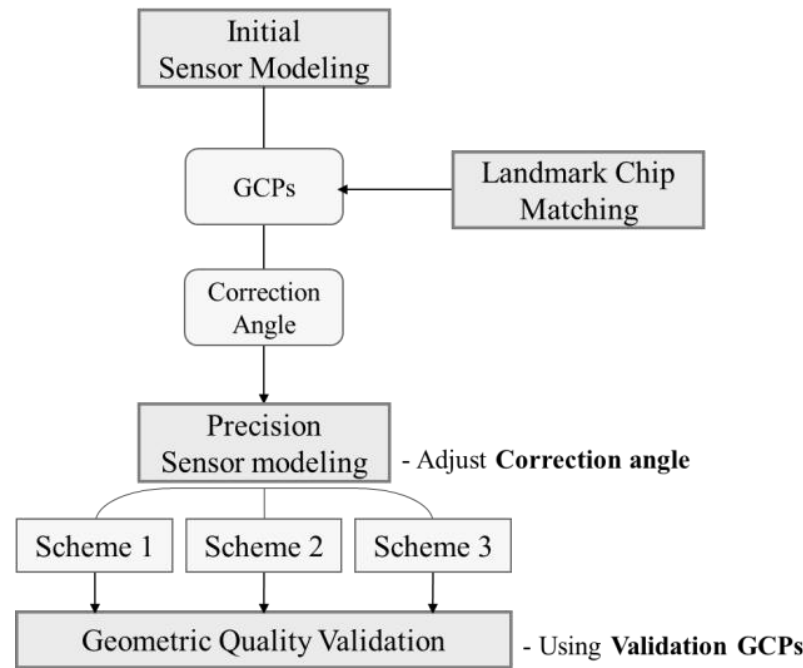

Figure 5. Quality comparison process

Overall process for comparing the quality of the three geometric correction schemes is shown in Figure 5.

Firstly, using ephemeris metadata of GOCI Level 1A, we established initial sensor model. And then, by matching edge detected from GOCI slot images and shoreline landmark chips, we calculated correction angle of each slot. After then, we conducted precision sensor modeling.

In order to analyze the quality of each geometric correction result, we generated 52 validation GCPs by measuring precision ground coordinates of GOCI images using Google map. We calculated RMSE (Root Mean Square Error) between true ground coordinates and estimated ground coordinates that computed by each precision sensor model.

\section{EXPERIMENTS AND ANALYSIS}

\subsection{Experiment Data}

Experiment data used in this paper was 8 datasets of $\mathrm{GOCI}$ Level 1A, which were photographed from 0:00 to 7:00 UTC April 5, 2011. GOCI Level 1A is the data without geometric correction and the spatial resolution of each band is $500 \mathrm{~m}$.

Figure 6 shows the 0:00 UTC dataset. As shown in this figure, validation GCPs cannot be obtained for slots 13,14 , and 15 , so they are excluded from this experiment. Therefore, in the scheme 2, geometric correction of slot 16 performed with the correction angle of slot 9 that is the closest slot among the previous slots.
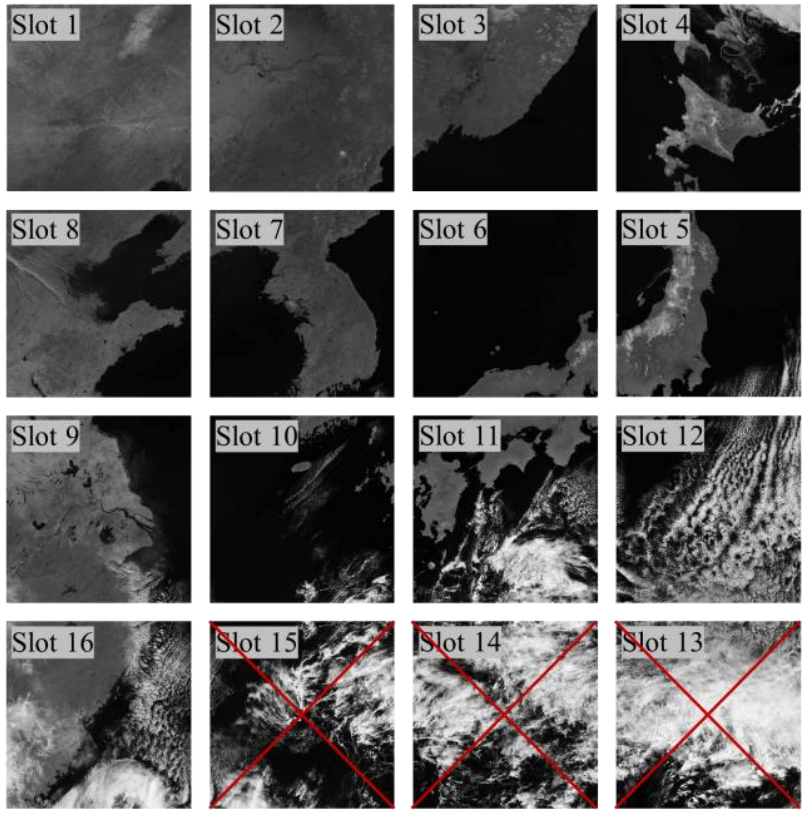

Figure 6. Experiment GOCI Slot image data

For shoreline matching, we used landmark database constructed by GSHHG (Global Self-consistent Hierarchical Highresolution Geography) shoreline database.

\subsection{Experiment Results}

Accuracy comparison of the three schemes in the all experiment GOCI Level 1A dataset was shown below.

\begin{tabular}{|c|c|c|c|c|}
\hline Slot & $\begin{array}{l}\text { Errors with } \\
\text { Slot Own } \\
\text { Correction } \\
\text { Angle } \\
(\mathrm{km})\end{array}$ & $\begin{array}{c}\text { Scheme } 1 \\
\text { Errors with } \\
\text { Previous } \\
\text { Time } \\
\text { Correction } \\
\text { Angle } \\
(\mathrm{km})\end{array}$ & $\begin{array}{c}\text { Scheme } 2 \\
\\
\text { Errors with } \\
\text { Previous } \\
\text { Slot } \\
\text { Correction } \\
\text { Angle } \\
(\mathrm{km})\end{array}$ & $\begin{array}{c}\text { Scheme } 3 \\
\text { Errors with } \\
\text { The Largest } \\
\text { Num of GCP } \\
\text { Slot } \\
\text { Correction } \\
\text { Angle } \\
(\mathrm{km})\end{array}$ \\
\hline 1 & $\begin{array}{l}3.0 \\
\end{array}$ & $\bar{~} 4.2$ & 2.8 & 2.7 \\
\hline 2 & 1.3 & 3.6 & 4.6 & 2.1 \\
\hline 3 & 1.2 & 3.6 & 1.4 & 1.9 \\
\hline 4 & 0.9 & 3.5 & 1.4 & 1.9 \\
\hline 5 & 1.1 & 3.3 & 0.8 & 1.5 \\
\hline 6 & 0.9 & 2.9 & 1.5 & 1.7 \\
\hline 7 & 0.7 & 2.8 & 0.7 & 2.1 \\
\hline 8 & 0.5 & 2.9 & 0.8 & 3.0 \\
\hline 9 & 1.2 & 3.0 & 0.8 & 2.8 \\
\hline 10 & 0.9 & 2.8 & 1.0 & 2.4 \\
\hline 11 & 1.0 & 2.6 & 1.3 & 2.3 \\
\hline 12 & 1.0 & 2.7 & 0.8 & 2.3 \\
\hline 16 & 1.5 & 3.7 & 1.0 & 2.7 \\
\hline Average & 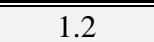 & $\begin{array}{c}3.2 \\
\end{array}$ & $\begin{array}{c}1.4 \\
\end{array}$ & 2.3 \\
\hline
\end{tabular}

Table 1. Accuracy comparison of the three schemes

Among three schemes, geometric correction applying the previous slot correction angle showed the best quality. The average RMSE of this scheme was about $1.4 \mathrm{~km}$, which was very close to geometric correction quality applying correction angles from GCPs. Error in scheme 1 were larger than that of scheme 2 . 
When applying the correction angle of the previous data, error increase was larger than applying the correction angle of the previous slot in almost every slot. This result showed that a variation of geometry between slots is smaller than that between datasets.

\begin{tabular}{|c|c|c|c|c|}
\hline \multirow[b]{2}{*}{ Slot } & 05:00 dataset & \multicolumn{3}{|c|}{ 06:00 dataset } \\
\hline & $\begin{array}{l}\text { Errors with } \\
\text { Slot Own } \\
\text { Correction } \\
\text { Angle } \\
(\mathrm{km})\end{array}$ & $\begin{array}{c}\text { Scheme } 1 \\
\text { Errors with } \\
\text { Previous } \\
\text { Time } \\
\text { Correction } \\
\text { Angle } \\
\text { (km) }\end{array}$ & $\begin{array}{c}\text { Scheme } 2 \\
\text { Errors with } \\
\text { Previous } \\
\text { Slot } \\
\text { Correction } \\
\text { Angle } \\
(\mathrm{km})\end{array}$ & $\begin{array}{c}\text { Scheme } 3 \\
\text { Errors with } \\
\text { The Largest } \\
\text { Num of GCF } \\
\text { Slot } \\
\text { Correction } \\
\text { Angle } \\
(\mathrm{km}) \\
\end{array}$ \\
\hline 1 & 1.4 & 7.0 & 1.4 & 1.8 \\
\hline 2 & 1.1 & 8.0 & 0.8 & 1.5 \\
\hline 3 & 1.1 & 7.9 & 0.8 & 1.4 \\
\hline 4 & 0.8 & 7.6 & 1.7 & 1.4 \\
\hline 5 & 1.3 & 6.7 & 0.7 & 0.9 \\
\hline 6 & 0.7 & 5.8 & 1.0 & 1.0 \\
\hline 7 & 0.8 & 6.2 & 0.9 & 1.4 \\
\hline 8 & 0.6 & 6.8 & 0.8 & 2.1 \\
\hline 9 & 1.0 & 5.2 & 0.6 & 1.5 \\
\hline 10 & 0.7 & 6.2 & 0.6 & 1.8 \\
\hline 11 & 0.9 & 6.2 & 1.6 & 1.7 \\
\hline 12 & 0.9 & 6.0 & 0.9 & 1.4 \\
\hline 16 & 0.9 & 5.1 & 2.1 & 1.7 \\
\hline Average & 0.9 & 6.5 & 1.1 & 1.5 \\
\hline
\end{tabular}

Table 2. Accuracy comparison of the three schemes in 6:00 data

For some datasets, the variation of geometry between datasets was so large that the geometric correction accuracy of the scheme 1 became very poor. Table 2 is an accuracy comparison of the three schemes in 6:00 dataset. Although the correction angle in the 05:00 dataset showed good performance, the result of scheme 1 in the 06:00 dataset, which reused this correction angle, showed low accuracy. On the other hand, results of scheme 2 and 3 showed better and more stable accuracy.

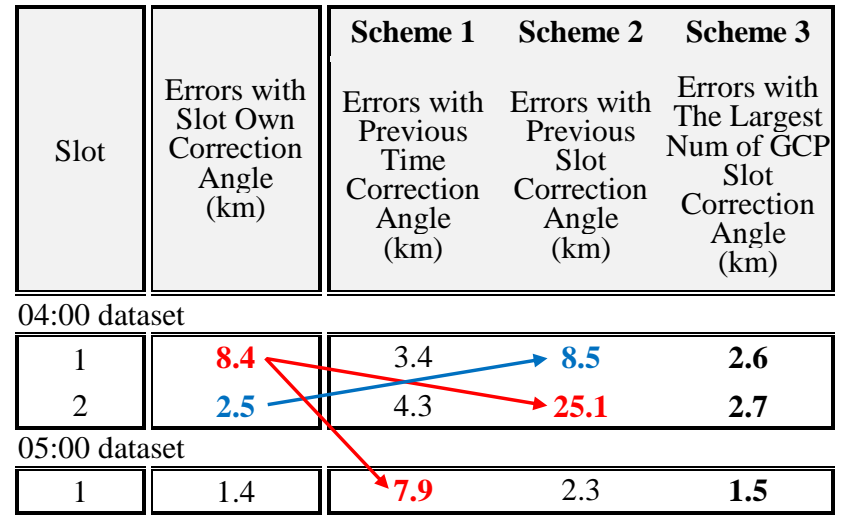

Table 3. Error propagation of the three schemes

In many cases of slot 1 and 2, scheme 3 showed the better quality than other schemes. Due to the small number of GCPs in slot 1 and 2, the performance of the correction angle was mostly poor. As shown in Table 3, error propagation increased when reusing a correction angle with poor performance. Therefore, if the previous time or slot correction angle performance is poor, scheme 3 showed better accuracy than other schemes.

\section{CONCLUSIONS}

In this paper, we proposed the geometric correction schemes reusing correction angle. Applying correction angle of the previous slot in the same time data was the best scheme for the geometric correction in slots without GCPs on average. However, if the previous slot correction angle performance is poor, applying correction angle of the slot with the largest number of GCPs in the same time data showed better accuracy than other schemes.

In the schemes tested, the error propagation is a major factor in the accuracy of geometric correction. Therefore, in future research, we will analyze a pattern of correction angle and reduce the error propagation. Furthermore, we will conduct geometric correction with frequency matching and compare the geometric correction quality with the results of this paper.

\section{ACKNOWLEDGEMENTS}

This research was supported by the "Development of the integrated data processing system for GOCI-II" funded by the Ministry of Ocean and Fisheries, Korea.

\section{REFERENCES}

Kim, H.G., 2018. Development of Geometric Correction Technique for Geostationary Ocean Color Imager, Master's Thesis, Inha University, Incheon, Korea, pp.1-27.

Yang, C.S., Song, J.H., 2012. Geometric performance evaluation of the Geostationary Ocean Color Imager. Ocean Sci $J$ (in this issue), pp. 235-246.

Lee, T.Y., Kim, T., Choi, H.J., 2005. Automated Landmark Extraction based on Matching and Robust Estimation with Geostationary Weather Satellite Images, Korean Journal of Remote Sensing, pp. 505-516 